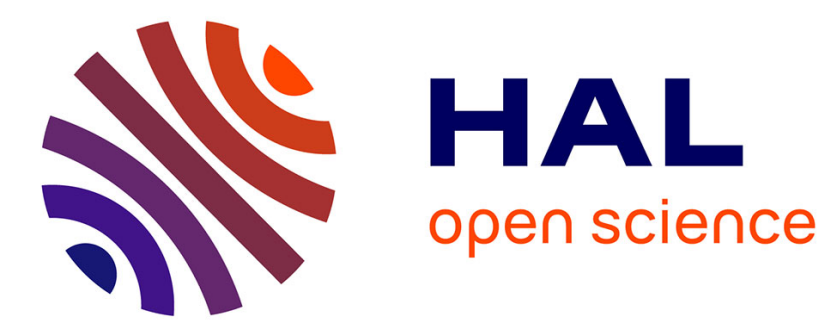

\title{
Phase imaging and synthetic aperture super-resolution via total internal reflection microscopy
}

Guillaume Maire, Hugues Giovannini, Anne Talneau, Patrick C. Chaumet, Kamal Belkebir, Anne Sentenac

\section{- To cite this version:}

Guillaume Maire, Hugues Giovannini, Anne Talneau, Patrick C. Chaumet, Kamal Belkebir, et al.. Phase imaging and synthetic aperture super-resolution via total internal reflection microscopy. Optics Letters, 2018, 43 (9), pp.2173. 10.1364/ol.43.002173 . hal-02023424

\section{HAL Id: hal-02023424 https://hal.science/hal-02023424}

Submitted on 18 Feb 2019

HAL is a multi-disciplinary open access archive for the deposit and dissemination of scientific research documents, whether they are published or not. The documents may come from teaching and research institutions in France or abroad, or from public or private research centers.
L'archive ouverte pluridisciplinaire HAL, est destinée au dépôt et à la diffusion de documents scientifiques de niveau recherche, publiés ou non, émanant des établissements d'enseignement et de recherche français ou étrangers, des laboratoires publics ou privés. 


\title{
Phase imaging and synthetic aperture super- resolution via total internal reflection microscopy
}

\author{
Guillaume Maire, ${ }^{1, *}$ Hugues Giovannini, ${ }^{1}$ Anne Talneau, ${ }^{2}$ Patrick C. Chaumet, ${ }^{1}$ Kamal Belkebir, \\ and Anne Sentenac ${ }^{1}$ \\ ${ }^{1}$ Aix Marseille University, CNRS, Centrale Marseille, Institut Fresnel, Marseille, France \\ ${ }^{2} \mathrm{CNRS/C2N}$, Centre de Nanosciences et de Nanotechnologies, Marcoussis, France \\ *Corresponding author: guillaume.maire@fresnel.fr
}

Received 6 February 2018; revised 30 March 2018; accepted 31 March 2018; posted 5 April 2018 (Doc. ID 321235); published 27 April 2018

\begin{abstract}
Total internal reflection microscopy is mainly used in its fluorescence mode and is the reference technique to image fluorescent proteins in the vicinity of cell membranes. Here, we show that this technique can easily become a phase microscope by simply detecting the coherent signal resulting from the interference between the field scattered by the probed sample and the total internal reflection. Moreover, combining several illumination angles permits generating synthetic aperture reconstructions with improved resolutions compared to standard label-free microscopy techniques. (๑) 2018 Optical Society of America
\end{abstract}

OCIS codes: (180.3170) Interference microscopy; (100.6640) Superresolution; (170.3880) Medical and biological imaging; (090.1995) Digital holography.

https://doi.org/10.1364/OL.43.002173

Total internal reflection fluorescence (TIRF) microscopy is the reference technique to study the membrane dynamics and organization in biological cells $[1,2]$. Its main advantage is the ability to illuminate with a high axial sectioning the sample, typically over a thin slice of about $100 \mathrm{~nm}$, by taking benefit from a total internal reflection (TIR) configuration. The sample is deposited on a glass substrate, and by illuminating through the substrate above the critical angle, an evanescent wave is created at the interface to locally probe the sample. Compared to standard wide-field fluorescence microscopy, images with highly improved contrast are provided, since the background signal stemming from the volume of the sample can be suppressed. The preferred TIRF configuration nowadays uses a high numerical aperture (NA) immersion objective both to illuminate above the critical angle and collect the fluorescence signal.

Using fluorophores as contrast agents, however, presents some drawbacks, as their addition is invasive and induces the increased risk of photobleaching and phototoxicity to the cell [3]. Long-term studies are thus difficult to achieve. The specificity of labeling is, moreover, at the same time the main advantage and the main limitation of fluorescence imaging, since the lack of structural images can be detrimental to the interpretation of the results. Therefore, there is a clear need for a high-resolution technique able to probe cell membranes without the constraint of incorporating a fluorescent label.

Two main approaches have been used so far to perform label-free imaging in TIR with a high NA objective. The first approach uses a spatially incoherent source (such as a light bulb or LED) to illuminate the sample simultaneously with all angles above the critical value imposed by the glass substrate and the sample immersion medium (usually water) [4,5]. As the sample refractive index is higher than that of water, TIR is frustrated at locations where the sample is close to the interface, which modulates the reflected intensity and is the source of contrast in the resulting image. The second approach uses a laser beam focused in the back focal plane (BFP) of the objective at the edge of the NA $[6,7]$. The focused beam is scanned along a circle in the $\mathrm{BFP}$, so that the sample is illuminated in TIR by a rotating collimated beam, and the integration time of the camera is set to match the rotation period of the beam. The totally reflected beam is blocked in a plane conjugated with the BFP to detect only the scattered intensity and obtain dark-field imaging. Both approaches have a high temporal resolution, and the second one claims an improved resolution at the price of some sample distortions in the image.

In this Letter, we propose a label-free TIR microscopy technique that keeps the same setup simplicity as the previous approaches, but permits besides to perform phase imaging and improve the resolution through synthetic aperture generation. The core idea of the technique is that the intensity detected in TIR microscopy can directly give access to the phase and the amplitude of the field scattered by the probed sample. Assuming the scalar approximation, the detected intensity $I$ can be written as

$$
I=\left|E_{r}+E_{s}\right|^{2}=\left|E_{r}\right|^{2}+\left|E_{s}\right|^{2}+E_{r} E_{s}^{*}+E_{r}^{*} E_{s},
$$

where $E_{r}$ is the field reflected by the interface in TIR, and $E_{s}$ is the field backscattered by the sample. As in TIR the sample is illuminated over a very thin slice and is usually of very weak contrast in biology, we can assume $E_{r}$ is very strong compared to $E_{s}$, and neglect $\left|E_{s}\right|^{2}$ in Eq. (1). Since $E_{r}$ is close to the edge 


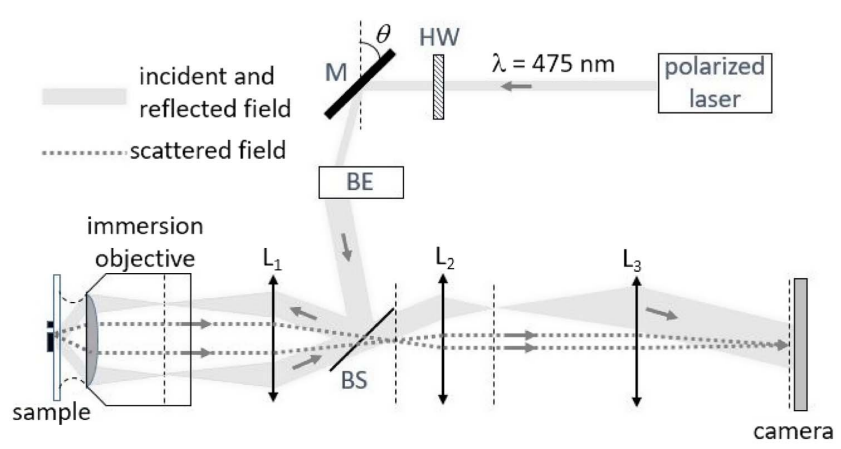

Fig. 1. Experimental setup: HW, motorized half-wave plate; $M$, rotative mirror; $\mathrm{BE}$, beam expander; $\mathrm{BS}$, beam splitter; $\mathrm{L}_{1}$, tube lens; $\mathrm{L}_{2,3}$, relay lenses.

of the NA of the objective, it can then act as an off-axis reference wave for $E_{s}$, as in classical digital holography $[8,9]$.

The experimental setup used to test this approach is sketched in Fig. 1. The light source is a supercontinuum laser (NKT Photonics SuperK Extreme EXW-12) filtered at $475 \mathrm{~nm}$ with a spectral width of $6 \mathrm{~nm}$ thanks to a variable bandpass filter (NKT Photonics SuperK Varia). The laser beam is linearly polarized and crosses a motorized half-wave plate (HW) to adjust the polarization direction. A fast-steering mirror ( $\mathrm{M}$, Newport FSM-300) permits to control the deflection of the beam, and a beam expander (BE) generates a wide collimated beam. The center of the mirror $\mathrm{M}$ is conjugated with the center of the sample through the $\mathrm{BE}$, the tube lens $\mathrm{L}_{1}$, and the microscope objective. Thus, rotating the mirror varies the illumination angle without shifting laterally the beam on the object. TIR illumination of the sample and collection of the reflected intensity are performed through the microscope objective (Nikon CFI Apo TIRF 100×, NA = 1.49). The signal is imaged on a sCMOS camera (Andor Zyla) after passing through relay lenses $L_{2}$ and $L_{3}$ to obtain a global magnification of about 290. The HW is rotated so that the polarization of the illumination remains parallel to the TIR interface for each illumination angle.

The setup was used on calibrated resin samples deposited on a glass slide. We present here the results obtained on a resin star shown in Fig. 2(a). The inner diameter of the star is $800 \mathrm{~nm}$, and each of its 12 branches has a width of $90 \mathrm{~nm}$ and a length of $400 \mathrm{~nm}$. Height of the sample is $160 \mathrm{~nm}$. The resin star was illuminated in TIR along six directions of polar angle about $60^{\circ}$, and with azimuthal angles regularly spaced every $60^{\circ}$. Figure 2(b) shows $I$ for the first illumination, and Fig. 2(c) its 2D Fourier transform (FT). The two circles appearing in Fig. 2(c) correspond to the FT of $E_{s}$ and $E_{s}^{*}$, each of them contained in the circular spatial frequency domain imposed by the NA of the objective. The central specular spot corresponds to the FT of $\left|E_{r}\right|^{2}$. As expected, $\left|E_{s}\right|^{2}$ is too weak to be distinguished, and thus $E_{s}$ and $E_{s}^{*}$ are clearly separated in the Fourier space, so that $E_{s}$ can be filtered [see Fig. 2(d)] and retrieved in phase and amplitude as in off-axis holography. Note that for the present sample, the contrast between resin and air is far larger than for biological samples in water; therefore, the condition $\left|E_{s}\right| \ll\left|E_{r}\right|$ will be still more valid in this case.

Once $E_{s}$ is filtered in the Fourier space for each illumination angle, it is possible to perform a synthetic aperture reconstruction
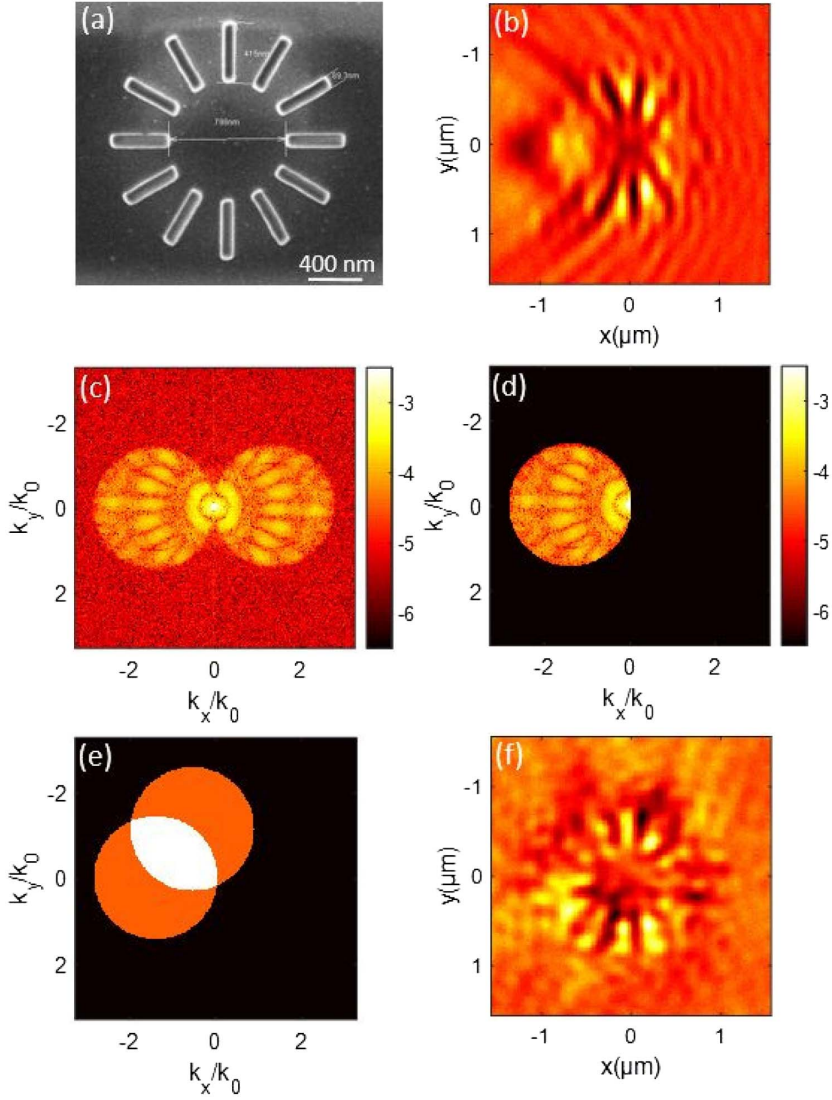

Fig. 2. (a) Electron microscope image of the sample: resin rods deposited on a glass slide; (b) detected intensity $I$ for the first TIR illumination angle; (c) 2D Fourier transform of (b), modulus shown in $\log$ scale, as a function of spatial frequencies $k_{x}$ and $k_{y}$, normalized with respect to wavevector modulus $k_{0}=2 \pi / \lambda$; (d) filtering of the scattered field contained in (c); (e) accessible spatial frequency domains for the first and second TIR illuminations, with common spatial frequencies shown in white; and ( $\mathrm{f}$ ) sum of $I$ for the six TIR illumination angles.

of the sample $[10,11]$. As it is illuminated over a very thin slice, a $2 \mathrm{D}$ model can be assumed, and the synthetic aperture can provide an equivalent reflectivity $r_{\mathrm{eq}}$ of the sample with a resolution beyond the Rayleigh criterion $[12,13]$. According to this model, the field scattered along wave vector $\mathbf{k}_{s}$ for an illumination along wave vector $\mathbf{k}_{i}$ can be written as

$$
E_{s}\left(\mathbf{k}_{s}, \mathbf{k}_{i}\right) \propto \tilde{r}_{\mathrm{eq}}\left(\mathbf{k}_{s, \|}-\mathbf{k}_{i, \|}\right),
$$

where $\mathbf{k}_{s, \|}$ and $\mathbf{k}_{i, \|}$ are the transverse components of $\mathbf{k}_{s}$ and $\mathbf{k}_{i}$, respectively, and $\tilde{r}_{\text {eq }}$ is the $2 \mathrm{D}$ FT of $r_{\text {eq }}$. As the signal is selfreferenced with $E_{r}, E_{s}$ is automatically correctly placed in the Fourier space to retrieve the spatial frequencies of $r_{\mathrm{eq}}$ [see Fig. 2(d)]. The synthetic aperture can thus in principle be generated simply from the sum of the fields for each illumination.

In practice, however, this is not that simple. For a given angle, the phase of the scattered field is retrieved at a given phase constant, which is the phase of $E_{r}^{*}$ for this particular angle, as seen in Eq. (1). In theory, the same polar angle is used for each illumination: this phase constant should remain the same for all the scattered fields, and the latter can be directly added to form the complex super-resolved image of the sample. But in practice 
this is never perfectly the case, and, moreover, the phase of $E_{r}$ is usually corrupted by the aberrations of the objective, which are particularly important at the edge of the NA. As a result, the phase of the scattered field is polluted with a different unknown value for each illumination. This effect can be seen by simply adding the different intensities $I$ for each illumination angle. In theory, it should provide the real part of $r_{\text {eq }}$ (as $E_{s}$ has not yet been filtered from $\left.E_{s}^{*}\right)$. This sum is displayed in Fig. 2(f), and shows an image distorted by the aberrations.

This issue can be solved by taking benefit from the spatial frequency overlap between the illuminations [12]. According to Eq. (2), two neighbor illumination angles give access to common spatial frequencies of $r_{\text {eq }}$, for which the scattered field should be the same in both cases. For example, Fig. 2(e) shows in white the common spatial frequency domain between illuminations 1 and 2. Thus, each illumination has been normalized with respect to its neighbor by multiplying its field by a complex factor that minimizes the discrepancy of the data in their common spatial frequency domain. This can be done, for instance, using standard minimization procedures implemented in MATLAB, applied to the function $F$ :

$$
F\left(u_{m}, v_{m}\right)=\sum_{\left(k_{x}, k_{y}\right) \in \Omega_{m}}\left|\tilde{E}_{s}^{m}\left(k_{x}, k_{y}\right)-u_{m} e^{i v_{m}} \tilde{E}_{s}^{m+1}\left(k_{x}, k_{y}\right)\right|,
$$

where $\tilde{E}_{s}^{m}$ is the $2 \mathrm{D}$ FT of the scattered field for the $m$ th illumination (as in Fig. 2(d) for illumination 1), $\Omega_{m}$ is the common spatial frequency domain between the $m$ th and $(m+1)$ th illuminations [as shown in white between illuminations 1 and 2 in Fig. 2(e)], and $u_{m}$ and $v_{m}$ are, respectively, the modulus and the argument of the complex factor to find. Figure 3 shows the building of the synthetic aperture by adding in Fourier space the filtered scattered field for the six illumination angles, with and without the normalization procedure. Aberrations clearly corrupt the synthetic aperture [Figs. 3(a) and 3(c)], and a striking improvement is brought by the normalization procedure [Figs. 3(b) and 3(d)].
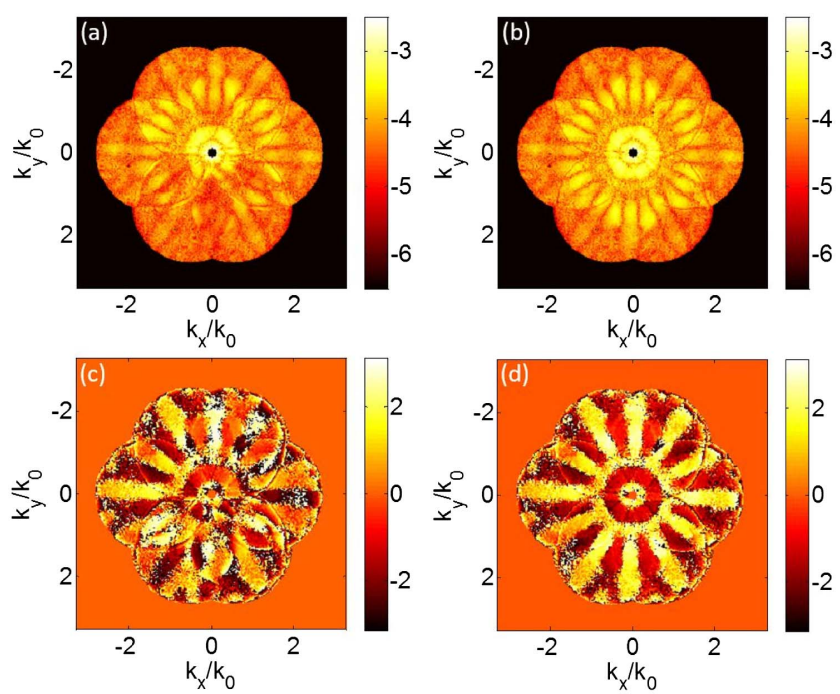

Fig. 3. (a) and (b) Modulus of the synthetic aperture before and after the normalization procedure, respectively (log scale, central specular spot has been removed to be in dark-field mode); and (c) and (d) same for the phase of the synthetic aperture. $r_{\text {eq }}$ can then be reconstructed in modulus and phase by doing the 2D inverse FT of the synthetic aperture. Figure 4(c) presents the squared modulus (intensity reflectivity) and Fig. $4(\mathrm{~d})$ the phase of $r_{\text {eq }}$. To improve the image contrast, Fig. 4(c) has been obtained in dark-field mode by canceling the central specular spot in Fourier space, as shown in Fig. 3(b). The size of the central black spot where data are suppressed is imposed by the field of view on the camera, and as it removes only the lowest spatial frequencies of the signal, it does not deteriorate the resolution of the reconstructions. On the other hand, to obtain the phase shift induced by the sample with respect to the background, Fig. 4(d) has been obtained in bright-field mode. It can be seen that Figs. 4(c) and 4(d) provide intensity and phase information with a clearly improved resolution compared to the intensity image obtained at normal incidence illumination [Fig. 4(a)], or by adding the intensities of the scattered fields for each TIR illumination angle [Fig. 4(b), dark-field image], as done in Refs. [6,7].

This resolution improvement is also striking when comparing Figs. 4(e) and 4(f), which plot the signal profile along the same white dashed circle for the dark-field image of Fig. 4(b) and the equivalent intensity reflectivity of Fig. 4(c), respectively.
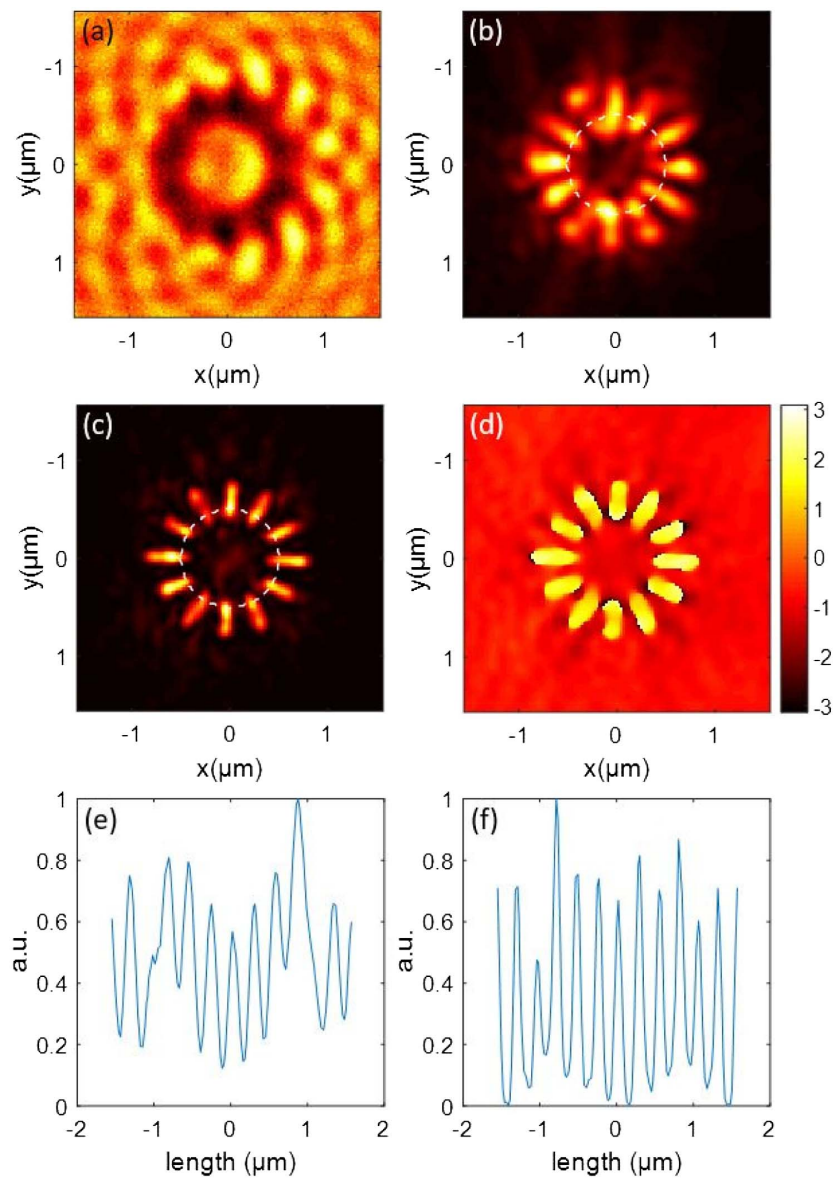

Fig. 4. (a) Intensity detected at normal incidence, in non-TIR mode; (b) dark-field image obtained by adding the intensities of the scattered field with filtered-out specular reflection for each TIR illumination angle; (c) intensity of the synthetic aperture reconstruction in dark-field mode; (d) phase of the synthetic aperture reconstruction in bright-field mode; and (e) and (f) plot of (b) and (c) along the white dashed circle, respectively. 
The 12 branches of the star are resolved with a high contrast in Fig. 4(f), whereas only 10 branches are retrieved in Fig. 4(e) and with a lower contrast. The separation distance between the branches at the center of the star is about $130 \mathrm{~nm}$, clearly below the Rayleigh criterion of about $200 \mathrm{~nm}$ imposed by the objective. Our reconstruction technique is presently run in less than five seconds on a standard laptop, but we believe this time can be shortened below one second with program optimization, so the method is compatible with in vivo imaging.

In conclusion, we have shown that any TIRF microscopy setup can become a phase microscope by detecting the interference between the scattered field and the TIR, as in off-axis holography but without the need of any external reference wave. By using several azimuthal illumination angles, it is possible to perform a synthetic aperture reconstruction of the sample, which provides intensity and phase images with improved resolution compared to classical microscopy techniques plagued by the Rayleigh criterion. Moreover, retrieving both the intensity and the phase of the scattered field permits to study the probed samples with two different image contrasts, which is richer than using intensity-only information as conventionally done in non-fluorescent TIR microscopy. With an appropriate model, we believe this can lead to improved estimations of quantitative parameters such as axial dimensions and refractive index of the sample, as in tomographic diffractive microscopy $[14,15]$.

Funding. Centre National de la Recherche Scientifique (CNRS).
Acknowledgment. The authors thank the "Mission pour l'Interdisciplinarité" of CNRS for its financial support.

\section{REFERENCES}

1. D. Axelrod, J. Cell Biol. 89, 141 (1981).

2. M. Brunstein, M. Teremetz, K. Hérault, C. Tourain, and M. Oheim, Biophys. J. 106, 1020 (2014).

3. C. M. Brown, J. Cell Sci. 120, 1703 (2007).

4. G. Byrne, M. Pitter, J. Zhang, F. Falcone, S. Stolnik, and M. Somekh, J. Microsc. 231, 168 (2008).

5. P. Bon, T. Barroca, S. Lévèque-Fort, and E. Fort, J. Biophoton. 7, 857 (2014).

6. P. von Olshausen and A. Rohrbach, Opt. Lett. 38, 4066 (2013).

7. F. Jünger, P. V. Olshausen, and A. Rohrbach, Sci. Rep. 6, 30393 (2016).

8. U. Schnars and W. Jüptner, Appl. Opt. 33, 179 (1994).

9. E. Cuche, P. Marquet, and C. Depeursinge, Appl. Opt. 38, 6994 (1999).

10. V. Lauer, J. Microsc. 205, 165 (2002).

11. Y. Cotte, F. Toy, P. Jourdain, N. Pavillon, D. Boss, P. Magistretti, P. Marquet, and C. Depeursinge, Nat. Photonics 7, 113 (2013).

12. V. Mico, Z. Zalevsky, P. Garcia-Martinez, and J. Garcia, J. Opt. Soc. Am. A 23, 3162 (2006).

13. S. A. Alexandrov, T. R. Hillman, T. Gutzler, and D. D. Sampson, Phys. Rev. Lett. 97, 168102 (2006).

14. C. Godavarthi, T. Zhang, G. Maire, P. C. Chaumet, H. Giovannini, A. Talneau, K. Belkebir, and A. Sentenac, J. Opt. Soc. Am. A 32, 287 (2015).

15. B. Simon, M. Debailleul, M. Houkal, C. Ecoffet, J. Bailleul, J. Lambert, A. Spangenberg, H. Liu, O. Soppera, and O. Haeberlé, Optica 4, 460 (2017). 